\title{
Hydrodynamics of Hemostasis in Sickle-Cell Disease
}

\section{Citation}

Cohen, S. I. A., and L. Mahadevan. 2013. "Hydrodynamics of Hemostasis in Sickle-Cell Disease." Physical Review Letters 110 (13). https://doi.org/10.1103/physrevlett.110.138104.

\section{Permanent link}

http://nrs.harvard.edu/urn-3:HUL.InstRepos:41417331

\section{Terms of Use}

This article was downloaded from Harvard University's DASH repository, and is made available under the terms and conditions applicable to Other Posted Material, as set forth at http:// nrs.harvard.edu/urn-3:HUL.InstRepos:dash.current.terms-of-use\#LAA

\section{Share Your Story}

The Harvard community has made this article openly available.

Please share how this access benefits you. Submit a story.

\section{Accessibility}




\title{
Hydrodynamics of Hemostasis in Sickle-Cell Disease
}

\author{
S. I. A. Cohen ${ }^{1, *}$ and L. Mahadevan ${ }^{1,2, \dagger}$ \\ ${ }^{1}$ School of Engineering and Applied Sciences, Harvard University, Cambridge, Massachusetts 02138, USA \\ ${ }^{2}$ Department of Physics, Department of Organismic and Evolutionary Biology, \\ Harvard University, Cambridge, Massachusetts 02138, USA
}

(Received 15 September 2012; published 28 March 2013)

\begin{abstract}
Vaso-occlusion, the stoppage of blood flow in sickle-cell disease, is a complex dynamical process spanning multiple time and length scales. Motivated by recent ex vivo microfluidic measurements of hemostasis using blood from sickle-cell patients, we develop a multiphase model that couples the kinetics and hydrodynamics of a flowing suspension of normal and sickled cells in a fluid. We use the model to derive expressions for the cell velocities and concentrations that quantify the hydrodynamics of hemostasis, and provide simple criteria as well as a phase diagram for occlusion, consistent with our simulations and earlier observations.
\end{abstract}

PACS numbers: 87.18.Nq, 87.19.rh, 87.19.U-, 87.19.X-

Sickle-cell disease (SCD) was the first genetic disease to be linked to the mutation of a specific protein [1]. The disease is caused by a point mutation in the molecule hemoglobin, the active constituent of red blood cells (RBCs) that transports oxygen from the lungs to the rest of the body. Unlike the normal protein, hemoglobin $A$ $(\mathrm{HbA})$, the mutant protein, hemoglobin $S$ ( $\mathrm{HbS})$, polymerizes to form filaments [2-4] under deoxygenated conditions typical of the venous system, a process that is reversible upon oxygenation in the lungs. The resulting $\mathrm{HbS}$ polymers alter the rigidity of RBCs and can lead to the characteristic sickled cell shape associated with the disease $[5,6]$. The stiffer RBCs slow down in the venous system where further oxygen starvation exacerbates the effect, eventually leading to vaso-occlusion, clogging, and hemostasis resulting in a crisis [7].

The events that lead to vaso-occlusion span multiple time and length scales $[8,9]$, from $\mathcal{O}\left(10^{-1} \mathrm{~s}\right)$ to $\mathcal{O}\left(10^{3} \mathrm{~s}\right)$ for the kinetics of $\mathrm{HbS}$ polymerization to the hydrodynamics of blood flow, and from $\mathcal{O}\left(10^{-9} \mathrm{~m}\right)$ to $\mathcal{O}\left(10^{-5} \mathrm{~m}\right)$ for the size of the protein to the dimensions of the circulatory vessels. In addition, the positive feedback between cell sickling and fluid flow makes the process both nonlinear and stochastic. While extensive work has been carried out to probe the individual determinants of SCD [5,10-13], integrating across these in order to provide quantitative insights into the overall dynamics of jamming in SCD remains a challenge. Here, we develop a minimal framework that attempts to capture the salient features of occlusion dynamics in terms of the chemical and physical processes at hand.

Recent studies of vaso-occlusion in SCD ex vivo $[9,14]$ capture the pathophysiology of this molecular disease while providing a simple biophysical assay for its likelihood. In these experiments, whole blood from patients is flowed through a microfluidic channel adjacent to a gas reservoir with permeable walls. Rapid deoxygenation (oxygenation) of the channel driven by the gas reservoir leads to occlusion (rescue). Figure 1 shows a sample of the dynamics of occlusion obtained as consecutive video frames [14] at the beginning [free flow, Fig. 1(a)] and end [occluded, Fig. 1(b)] of the process. We see that occlusion corresponds to the transition from the unimpeded flow of a soft suspension of cells which move relative to one another [Fig. 1(a)] to the formation of a porous plug of cells through which plasma may continue to flow [Fig. 1(b)].

To understand this, we consider the flow of blood, initially containing plasma and unsickled cells, through a capillary channel. The entire channel is rapidly and homogeneously deoxygenated at time $t=0$ and maintained in this state [14], with plasma and unsickled cells continuing to flow in from the boundary at $x=0$, Fig. 1(c). As in the experiments $[9,14]$, we assume a constant pressure drop over the deoxygenated channel through the entire process. To quantify the dynamics of occlusion, we choose to describe this system in terms of the flow of a multiphase fluid where the volume fractions of plasma, sickled RBCs and unsickled RBCs are denoted as $\phi_{\circ}(t, \mathbf{x}), \phi_{+}(t, \mathbf{x})$, and $\phi_{-}(t, \mathbf{x})$, respectively, with $\phi_{\circ}+\phi_{+}+\phi_{-}=1$. The evolution of these fractions is described by fluid mass conservation, and the advection and the conversion of unsickled to sickled cells: $\partial \phi_{\circ} / \partial t+\nabla \cdot\left(\mathbf{u}_{\circ} \phi_{\circ}\right)=0, \partial \phi_{ \pm} / \partial t+\nabla \cdot$ $\left(\mathbf{u}_{ \pm} \phi_{ \pm}\right)= \pm \phi_{-} / \tau_{K}$, where the sickling is assumed to follow first-order kinetics with a characteristic kinetic time $\tau_{K}[5,8,15,16]$. Since RBCs are $4 \mu \mathrm{m}$ in radius, their equilibrium diffusivity is negligible, but their nonequilibrium effective hydrodynamic diffusivity is nonzero [17]; here we neglect this effect, focusing on a mean-field theory.

When few cells have sickled [Fig. 1(a)], the plasma and RBCs flow freely and we may describe the velocity of the plasma $\mathbf{u}_{\circ}$ and the cells $\mathbf{u}_{ \pm}\left(=\mathbf{u}_{+}=\mathbf{u}_{-}\right.$, assuming that the sickled and unsickled cells move at the same velocity) effectively using the Stokes approximation: $\nabla p \sim \eta_{0} \nabla^{2} \mathbf{u}_{\circ}$ with $\mathbf{u}_{ \pm}=\mathbf{u}_{\circ}$, and $\eta_{0}$ being an effective 


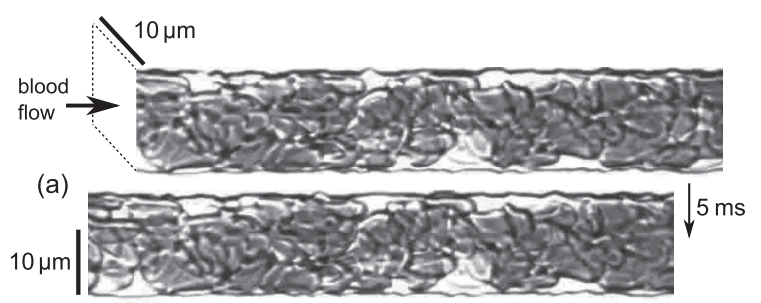

(b)
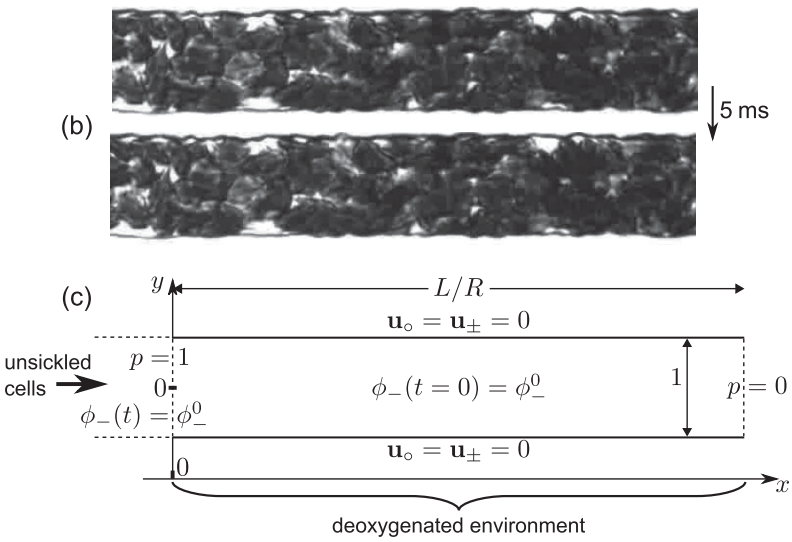

FIG. 1. Consecutive video frames (from movie S1 of Ref. [14]) of a section of the capillary channel (a) just after the oxygen concentration has been lowered, and (b) later after the channel has jammed. The images in (a) and (b) are translated to reflect the cell velocities. The increased darkness of the cells follows the deoxygenation of intracellular HbS [14]. (c) shows the scaled geometry and the initial and boundary conditions used in conjunction with (1)-(5).

suspension viscosity. As further cells sickle, the relatively unimpeded flow of the cells and plasma progressively transforms as the cells form a slowly moving porous plug through which the plasma continues to flow. This leads to a flow regime better described by the Darcy approximation for flow through porous media: $\nabla p \sim \eta_{0}\left(\mathbf{u}_{\circ}-\mathbf{u}_{ \pm}\right) / \kappa_{0}$, where $\kappa_{0}$ is the hydraulic permeability of the porous plug. Exploiting the linearity of the equations describing the two limits, we interpolate the transition from Stokes flow to Darcy flow by using the momentum balance equations of the form $\nabla p=\eta_{0}\left\{\nabla^{2} \mathbf{u}_{\circ}-\left(\mathbf{u}_{\circ}-\mathbf{u}_{ \pm}\right) / \kappa_{0}\right\}$; this form is similar to the Darcy-Brinkman equation [18]. To complete the formulation of the problem, we require a relationship between the plasma and cell velocities. At the cellular scale, the stiffening of RBCs due to intracellular $\mathrm{HbS}$ aggregation causes an increase in the strength and frequency of interactions with the vessel walls and with other cells $[19,20]$. The cell velocity starts to progressively differ from that of the plasma, and eventually, when a critical volume fraction of cells have sickled, the cell velocity falls to zero resulting in vaso-occlusion [Fig. 1(b)]. Neglecting the role of inertia, we describe the collective slowing down of the cells, due to their increasingly frequent interactions, using the local power-law relationship $\mathbf{u}_{\circ}-\mathbf{u}_{ \pm}=$ $\left(\phi_{+} / \phi_{*}\right)^{\gamma} \mathbf{u}_{\circ}$, where $\phi_{*}$ is the critical volume fraction of sickled cells at jamming, and $\kappa_{0} /\left(\phi_{+} / \phi_{*}\right)^{\gamma}$ is the effective permeability of the cells. This form recovers the two limits $\mathbf{u}_{ \pm}=\mathbf{u}_{\circ}$ when $\phi_{+}=0$, i.e., there are no sickled cells, and $\mathbf{u}_{ \pm}=0$ when $\phi_{+}=\phi_{*}$, i.e., at occlusion; the exponent $\gamma$ controls the sharpness of the transition.

For flow through a high-aspect ratio channel, Fig. 1(c), of length $L$ and height $R$ with an initial cell fraction (hematocrit) $\phi_{-}^{0}[\approx 0.25$ in Fig. 1(a)], we define the dimensionless quantities (indicated using tildes) via: $u=$ $v_{0} \tilde{u}$ with $v_{0}:=p_{0} R^{2} /\left(8 L \eta_{0}\right), t:=\tau_{H} \tilde{t}$ with $\tau_{H}:=$ $L / v_{0}=L^{2} \eta_{0} /\left(p_{0} R^{2}\right), p=p_{0} \tilde{p}$ with the pressure difference $p_{0}$, and $\mathbf{x}=x_{0} \tilde{\mathbf{x}}$ with $x_{0}=R$. Dropping the tildes from here on leads to the dimensionless equations

$$
\begin{gathered}
\partial \phi_{\circ} / \partial t+\epsilon^{-1} \nabla \cdot\left(\mathbf{u}_{\circ} \phi_{\circ}\right)=0, \\
\partial \phi_{ \pm} / \partial t+\epsilon^{-1} \nabla \cdot\left(\mathbf{u}_{ \pm} \phi_{ \pm}\right)= \pm \phi_{-} \tau_{H} / \tau_{K}, \\
\phi_{\circ}+\phi_{+}+\phi_{-}=1, \\
\nabla^{2} \mathbf{u}_{\circ}-\left(\phi_{+} / \phi_{\bullet}\right)^{\gamma} \mathbf{u}_{\circ}=8 \epsilon^{-1} \nabla p, \\
{\left[1-\left(\phi_{+} / \phi_{*}\right)^{\gamma}\right] \mathbf{u}_{\circ}=\mathbf{u}_{ \pm},}
\end{gathered}
$$

for the variables $\phi_{\circ}, \phi_{+}, \phi_{-}, \mathbf{u}_{+}, \mathbf{u}_{-}, \mathbf{u}_{\circ}, p$ where the aspect ratio $\epsilon^{-1}:=L / R \gg 1$ and we identify $\tau_{H} / \tau_{K}$ as the dimensionless ratio of a hydrodynamic time for transit through the capillary to a kinetic time for cell sickling, and $\phi_{.}:=\phi_{*}\left(\kappa_{0} / R^{2}\right)^{1 / \gamma}<\phi_{*}$ as a characteristic scaled volume fraction of sickled cells given by the ratio of the jamming fraction $\phi_{*}$ and the dimensionless permeability.

Interestingly, although the critical fraction $\phi_{*}$ defines the fraction of sickle cells at a blockage, the crossover in the rheology associated with the Stokes-Darcy transition according to (4) occurs at a lower crossover sickle-cell fraction $\phi_{+} \sim \phi_{.}$, when the cells are still mobile. As the sickle-cell fraction increases, positive feedback associated with the increased resistance to flow for a given pressure drop provides additional time for the fraction of sickled cells to increase, and the cell and plasma velocities deviate more and more according to (5). Eventually, the fluid velocity $\mathbf{u}_{\circ} \rightarrow-8 \epsilon^{-1}\left(\kappa_{0} / R^{2}\right)^{1 / \gamma} \nabla p$ from (4), and occlusion occurs as $\phi_{+} \rightarrow \phi_{*}$.

To build on this qualitative picture, we now analyze solutions to (1)-(5) computed for $2 \mathrm{D}$ flow using the COMSOL finite element package [21] and provide simple analytical estimates that help us to understand the various flow regimes.

No occlusion.-When few cells sickle during transit, the plasma and cell velocities remain approximately unchanged relative to those of the original Stokes flow, $\mathbf{u}_{S}:=\left(u_{S}, 0\right)$, and occlusion does not occur. The channel residence time $t_{o}$ for a fluid-cell parcel is approximately $t_{o}=x \epsilon u_{S}^{-1}$ for parcels that have flowed in at $t>0$, and $t_{o}=t$ for parcels that occupied the channel at $t=0$ but have yet to exit. Therefore, the sickle-cell fraction, which evolves through first-order kinetics, is $\phi_{+}=\phi_{-}^{0}[1-$ $\left.\exp \left(-\min \left(t, x \epsilon u_{S}^{-1}\right) \tau_{H} / \tau_{K}\right)\right]$, as shown in Fig. 2 and 

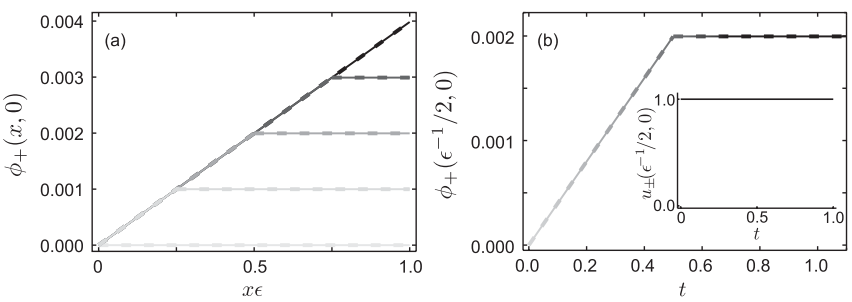

FIG. 2. Nonocclusive dynamics. (a) Sickle-cell fraction profiles along the channel for increasing times (gray to black) uniform from $t=0$ to $t=1$. The position of the knee corresponds to that of a fluid-cell parcel that was at the inlet at $t=0$ and has position $x \epsilon=u_{S} t$. The steady-state profile emerges when this parcel reaches the outlet. (b) Sickle-cell fraction in the center of the channel as a function of time. The inset shows the cell velocity, which is approximately constant at the initial value. In (a) and (b), the solid lines are numerical solutions to (1)-(5), with the initial and boundary conditions from Fig. 1(c); the dashed lines are the analytical solution (6) with $\bar{u}=u_{S}=$ $4(1 / 2-y)(1 / 2+y)$. The parameters were $\epsilon^{-1}=100, \gamma=2$, $\phi_{u}^{0}=0.5, \phi_{*}=0.7, \phi_{\bullet}=0.1, \tau_{H} / \tau_{K}=8 \times 10^{-3}$, yielding $\alpha=20$ from (10). See video 1 in the Supplemental Material [22] for the evolution throughout the channel.

video 1 in the Supplemental Material [22], and compared to numerical solutions to (1)-(5). Since the velocity $u_{S}$ remains constant in time, the second term in the minimum operator is the smallest for $t \gg u_{S}^{-1}$, implying that cells initially at the inlet have exited the channel, leading to the steady-state profile $\phi_{+}=\phi_{-}^{0}\left[1-\exp \left(-x \epsilon u_{S}^{-1} \tau_{H} / \tau_{K}\right)\right]$. Thus, although there is progressive sickling along the microchannel, too few cells sickle before reaching the outlet to effect significantly the bulk rheology of the suspension.

Occlusion.-If the sickle-cell fraction increases sufficiently, however, the cell and plasma velocities progressively deviate from those of the original flow profile. The channel residence time for a fluid-cell parcel is the sum of times taken to traverse each distance $d s, t_{o}=$ $\min \left(t, x \in \int_{0}^{x} u_{ \pm}(t(s), s)^{-1} d s / x\right) \equiv \min \left(t, x \in \bar{u}^{-1}\right)$, yielding

$$
\phi_{+}=\phi_{-}^{0}\left[1-\exp \left(-\min \left(t, x \epsilon \bar{u}^{-1}\right) \tau_{H} / \tau_{K}\right)\right]
$$

where $\bar{u}$ is an average longitudinal velocity for each parcel bounded by its initial and current velocities. The transition from nonocclusive to occlusive flow is shown in Fig. 3 and video 2 in the Supplemental Material [22]. In a Lagrangian framework, we see that cells starting at the inlet move initially at an unperturbed velocity $\mathbf{u}_{ \pm} \approx \mathbf{u}_{S}\left(\bar{u} \approx u_{S}\right)$ as they sickle [Figs. 2(a) and 3(a)]. In the nonocclusive case, they reach the outlet with the steady state concentration profile [Fig. 2(a)]. In contrast, if the sickle-cell fraction reaches $\phi_{+} \sim \phi$. during transit [Fig. 3(b)], the cell velocity $\mathbf{u}_{ \pm}$(and $\bar{u}$ ) decreases [Fig. 3(d)] and the sicklecell fraction increases [Fig. 3(c)] until the channel gets blocked. In contrast to nonocclusive dynamics, the first term in the minimum operator in (6) dominates for long
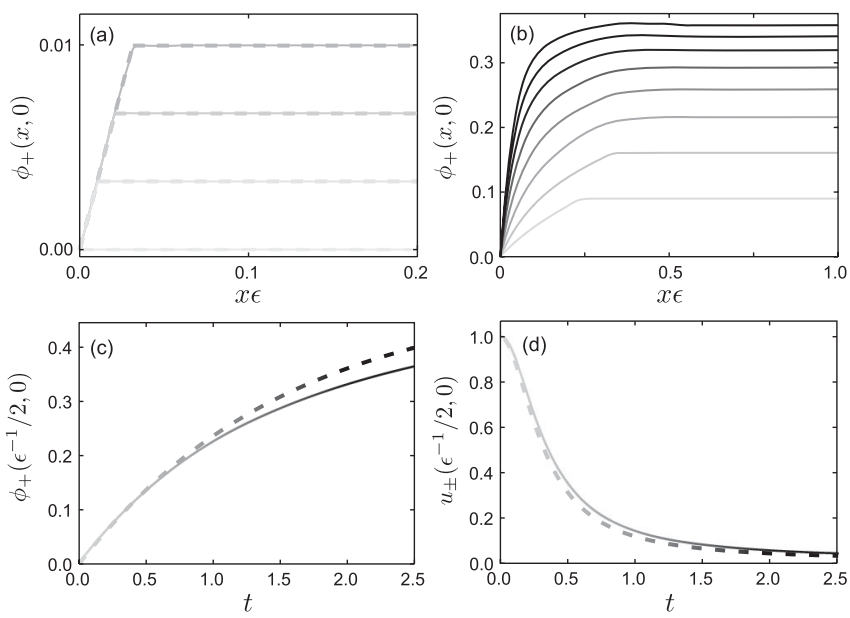

FIG. 3. Occlusive dynamics. (a) Sickle-cell fraction for early times from $t=0$ to $t=0.03$ (light to dark gray) before $\phi_{+}$ reaches $\phi_{\bullet}$, analogous to nonocclusive flow from Fig. 2(a). The position of the knee, initially $x \epsilon \approx u_{S} t$ around which the $x$ axis is zoomed, corresponds to that of a fluid-cell parcel which was at the inlet at $t=0$; in the occlusive case this parcel never reaches the outlet. The dashed lines are (6) with $\bar{u}=u_{S}$. (b) Sickle-cell fraction for later times, uniform from $t=0.3$ to $t=2.5$, where $\phi_{+}>\phi_{\bullet}$, showing gradual blockage of the channel as $\phi_{+}$ increases towards $\phi_{*}$. (c) Sickle-cell fraction at the center of the channel as a function of time. The dashed line is (7). (d) Cell velocity at the center of the channel showing the deceleration as occlusion occurs. The dashed line is the analytical result (5), (7), and (8). In (a)-(d), the solid lines are calculated numerically from (1)-(5) with the initial and boundary conditions from Fig. 1(c). The parameters were $\epsilon^{-1}=20, \gamma=2, \phi_{u}^{0}=0.5$, $\phi_{*}=0.7, \phi_{\bullet}=0.03, \tau_{H} / \tau_{K}=0.6$, yielding $\alpha=0.1$ from (10) and $\beta=0.04$, giving a characteristic time scale for occlusion $\alpha / \beta=2.5$. See video 2 in the Supplemental Material [22] for the evolution throughout the channel.

times, implying that cells initially at the inlet never exit the channel, and the sickle-cell fraction increases to $\phi_{*}$ with a concomitant occlusion.

Away from the inlet to the channel, (6) reduces to

$$
\phi_{+}=\phi_{-}^{0}\left[1-\exp \left(-t \tau_{H} / \tau_{K}\right)\right] .
$$

For the high aspect ratio capillary, where $\partial^{2} u / \partial x^{2} \ll$ $\partial u^{2} / \partial y^{2}$ and the longitudinal pressure gradient $\partial p / \partial x \approx$ $\epsilon$ is relatively uniform with $\partial p / \partial y \approx 0$, substituting the approximate velocity field $\mathbf{u}_{\circ}=\left(u_{\circ}(y), 0\right)$ into (4) yields $\partial^{2} u_{\circ} / \partial y^{2}-\left(\phi_{+} / \phi_{\bullet}\right)^{\gamma} u_{\circ}=-8$, subject to the boundary conditions $u_{\circ}( \pm 1 / 2)=0$. This system has the solution

$$
u_{\bullet}(y)=8\left(\phi_{\bullet} / \phi_{+}\right)^{\gamma}\left[1-\frac{\cosh \left(\left(\phi_{+} / \phi_{\bullet}\right)^{\gamma / 2} y\right)}{\cosh \left(\left(\phi_{+} / \phi_{\bullet}\right)^{\gamma / 2} / 2\right)}\right]
$$

for the plasma velocity in terms of the sickle-cell fraction, which together with (5) and (7) yields the cell velocity along the channel and compares well with results of numerical simulations of the full problem [Fig. 3(d)]. 
Using a first-order expansion of the exponential in (7) further simplifies the form of the longitudinal cell velocity $\mathbf{u}_{ \pm}=\left(u_{ \pm}(y), 0\right)$ yielding

$$
u_{ \pm}(y)=\underbrace{\left[1-\left(\frac{\beta t}{\alpha}\right)^{\gamma}\right]}_{d(t)} \underbrace{8\left(\frac{\alpha}{t}\right)^{\gamma}\left[1-\frac{\cosh \left((t / \alpha)^{\gamma / 2} y\right)}{\cosh \left((t / \alpha)^{\gamma / 2} / 2\right)}\right]}_{u_{\circ}(t)},
$$

with $\alpha:=\left(\tau_{K} / \tau_{H}\right)\left(\phi_{\bullet} / \phi_{-}^{0}\right), \beta:=\phi_{\bullet} / \phi_{*}=\left(\kappa_{0} / R^{2}\right)^{1 / \gamma}$, and valid up to $t<\alpha / \beta$, a characteristic time to occlusion. Here, $\alpha$ is the product of two factors: the ratio of the kinetic time to the hydrodynamic time and the ratio of the crossover fraction of sickled cells to the initial hematocrit, while $\beta$ is the ratio of the crossover sickle fraction at the Stokes-Darcy transition to the critical fraction at occlusion, or equivalently the hydraulic permeability $\kappa_{0}$ scaled by the channel width $R$.

Decomposing the cell velocity (9) into the plasma velocity $u_{\circ}(t)$ and the differential velocity between the plasma and cells $d(t)$ shows the crucial role of this cell-plasma differential velocity, driven by sickling. A comparison of our predictions with ex vivo experimental data for blood from a patient with severe SCD flowing through microchannels [14] is shown in Fig. 4(a); by fitting the parameters $\alpha, \beta$ in (9) we capture the experimentally observed flow deceleration curve until hemostasis.

Figure 4(b) shows a phase diagram for the dynamics of flow and stasis in SCD, characterized by the ratio of the kinetic to the hydrodynamic time scales and the scaled initial
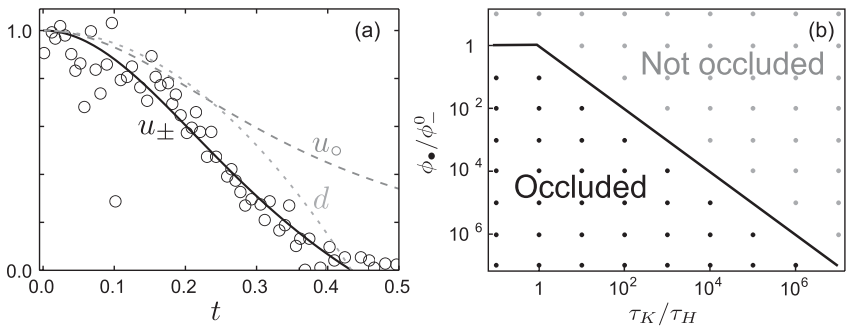

FIG. 4. (a) Two-parameter fit (solid line) of (9) to experimental data (open circles) for the RBC velocity as a function of time from ex vivo experiments using blood from a SCD patient [14]. The fit parameters are $\alpha=0.12, \beta=0.27$ with $\gamma=2$ [27], giving an occlusive time scale $\alpha / \beta=0.4$. The dashed line is the predicted velocity of the plasma phase, labeled $u_{\circ}(t)$ in (9), and the dotted line is the ratio of the cell and plasma velocities, labeled $d(t)$ in (9); the solid line is their product. The data are scaled using the mean $\mathrm{HbA}$ blood velocity $v_{0}=2.2 \times$

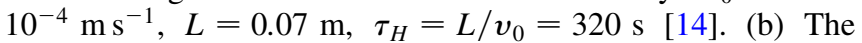
phase diagram in terms of the ratios of the kinetic to hydrodynamic time scales and the crossover sickle-cell fraction $\phi$. to the initial cell fraction $\phi_{-}^{0}$. The solid line is (10); the dots are numerical results from (1)-(5); gray $=$ not occluded, black $=$ occluded. cell fraction. Vaso-occlusion occurs when the bulk cell velocity is zero, a condition satisfied when $\phi_{+}$reaches $\phi$. in a time $t \lesssim 1$, which implies $\tau_{H} / \tau_{K}>-\log \left(1-\phi_{\bullet} / \phi_{-}^{0}\right)$. For $\phi_{\bullet} / \phi_{-}^{0}<1$, expanding the logarithm to first order yields the simple criteria

$$
\alpha=\frac{\tau_{K}}{\tau_{H}} \frac{\phi_{\bullet}}{\phi_{-}^{0}} \begin{cases}\gg 1 & \text { no occlusion } \\ \ll 1 & \text { occlusion, }\end{cases}
$$

which are intuitive: a small polymerization time scale $\tau_{K}$ for sickling or a long hydrodynamic time scale $\tau_{H}$ for traversing the microcirculation favors occlusion, as does a high hematocrit $\phi_{-}^{0}$ or a low crossover sickle fraction $\phi$. Thus, while the time scale for occlusion is controlled by $\alpha / \beta$, the condition for occlusion is controlled by $\alpha$.

Analysis of experimental data for flow deceleration, such as in Fig. 4(a), for five patients [14,23] suffering from severe SCD yields the ranges $0.10<\alpha<0.13$ and $0.27<\beta<0.39$, consistent with the phase diagram Fig. 4(b) and the criteria (10). Since $\tau_{H} \approx 320 \mathrm{~s}, \phi_{-}^{0} \approx 0.3$, and $0.3<\phi_{*}<1$ for these experiments [14,23], these values of $\alpha$ and $\beta$ imply $\tau_{K} \approx 60 \mathrm{~s}, \phi_{\bullet} \approx 0.2$ and an occlusive time scale of $\tau_{H} \alpha / \beta \approx 130 \mathrm{~s}$, consistent with hydrodynamic experiments $[9,14]$ and in agreement with in vitro $\mathrm{HbS}$ polymerization kinetics $[15,16]$.

Our work integrates the kinetics and hydrodynamics of capillary flow and hemostasis in SCD in terms of a minimal multiphase model with just two parameters, $\alpha$ and $\beta$, that quantify (i) the kinetic hypothesis in SCD [4,5,8,9,24,25], where vaso-occlusion is favored when the ratio of a characteristic time scale for cell sickling to that for the RBCs to traverse the microcirculation, $\tau_{K} / \tau_{H}$ in (10), is small, and (ii) the characteristic concentrations of cells, i.e., the initial hematocrit, scaled by a crossover concentration $\phi_{\bullet} / \phi_{-}^{0}$ in the condition for occlusion (10), and scaled by the critical sickle fraction $\phi_{*} / \phi_{-}^{0}$ in the occlusive time scale $\alpha / \beta$. The results are summarized by the simple criteria (10) and the accompanying phase diagram Fig. 4(b), both of which characterize occlusion.

Clinically, the primary determinant of disease severity in patients $[5,24]$ is likely to be the ratio $\tau_{K} / \tau_{H}$ in $\alpha$, consistent with our recent study distinguishing patients with benign and severe symptoms [14]. In addition, the differences in the characteristic concentrations, particularly $\phi_{\text {. }}$, might also contribute to the variations between patients with severe disease [14]. Going forward, it would be interesting to study the dissolution of jams upon reoxygenation when cells unsickle, and the observed hysteresis $[9,26]$; indeed, this follows from our model when the reaction term in (2) is reversed to read $\mp \phi_{+} \tau_{H} / \tau_{U}$, where $\tau_{U}<\tau_{K}[9]$ is the characteristic time for cells to unsickle. Our theory does not account for fluctuations; accounting for these via the incorporation of nonequilibrium diffusivity and the microscopic mechanisms by which cells stiffen and decelerate heterogeneously is crucial for a quantitative personalized diagnostic for hemostasis in SCD. 
We thank D. K. Wood, J.M. Higgins, L. Rajah, and T.P. J. Knowles for helpful discussions, and the Schiff Foundation (S.I.A.C.), the Kennedy Memorial Trust (S. I. A. C.), the Kavli Institute for Nanobio Science and Technology at Harvard (L.M.), and the MacArthur Foundation (L. M.) for support.

*Present address: Department of Chemistry, University of Cambridge, Lensfield Road, Cambridge, CB2 1EW, U.K. †m@seas.harvard.edu

[1] L. Pauling and H. A. Itano, Science 110, 543 (1949).

[2] F. A. Ferrone, J. Hofrichter, and W. A. Eaton, J. Mol. Biol. 183, 611 (1985).

[3] F. Ferrone, Methods Enzymol. 309, 256 (1999).

[4] C. T. Noguchi and A.N. Schechter, Blood 58, 1057 (1981).

[5] W. A. Eaton and J. Hofrichter, Adv. Protein Chem. 40, 63 (1990).

[6] G. W. Christoph, J. Hofrichter, and W. A. Eaton, Biophys. J. 88, 1371 (2005).

[7] M. H. Steinberg, N. Engl. J. Med. 340, 1021 (1999).

[8] F. A. Ferrone, Microcirculation 11, 115 (2004).

[9] J.M. Higgins, D. T. Eddington, S. N. Bhatia, and L. Mahadevan, Proc. Natl. Acad. Sci. U.S.A. 104, 20496 (2007).

[10] C. T. Noguchi, D. A. Torchia, and A. N. Schechter, Proc. Natl. Acad. Sci. U.S.A. 77, 5487 (1980).

[11] H. Hiruma, C. T. Noguchi, N. Uyesaka, A. N. Schechter, and G. P. Rodgers, Am. J. Physiol. 268, H2003 (1995).

[12] T. Itoh, S. Chien, and S. Usami, Blood 85, 2245 (1995).
[13] Z. Huang, L. Hearne, C. E. Irby, S. B. King, S. K. Ballas, and D. B. Kim-Shapiro, Biophys. J. 85, 2374 (2003).

[14] D. K. Wood, A. Soriano, L. Mahadevan, J. M. Higgins, and S. N. Bhatia, Sci. Transl. Med. 4, 123ra26 (2012).

[15] Z. Cao and F. A. Ferrone, Biophys. J. 72, 343 (1997).

[16] J. Hofrichter, J. Mol. Biol. 189, 553 (1986).

[17] J. M. Higgins, D. T. Eddington, S. N. Bhatia, and L. Mahadevan, PLoS Comput. Biol. 5, e1000288 (2009).

[18] H. C. Brinkman, Appl. Sci. Res. Sect. A 1, 27 (1949).

[19] J. M. Skotheim and L. Mahadevan, Phys. Rev. Lett. 92, 245509 (2004).

[20] This is due to a weakening of the elastohydrodynamic effect [19] that generates normal forces between sheared cells, allowing them to squeeze past each other. In vivo, attachment of cells to capillary walls, which does not occur in the microfluidic experiments, may also occur [14].

[21] COMSOL 4.2, Burlington, MA, USA, http://www.comsol .com.

[22] See Supplemental Material at http://link.aps.org/ supplemental/10.1103/PhysRevLett.110.138104 for videos showing the simulated evolution of $\phi_{+}(x, y)$ and $u_{ \pm}(x, y)$.

[23] Different pressures were used for each measurement [14]; to enable a comparison between patients, values of $\alpha$ have been scaled to correspond to $\tau_{H}=320 \mathrm{~s}$ as in Fig. 4(a).

[24] W. A. Eaton, J. Hofrichter, and P. D. Ross, Blood 47, 621 (1976).

[25] H. R. Sunshine, J. Hofrichter, and W. A. Eaton, Nature (London) 275, 238 (1978).

[26] W. A. Eaton and J. Hofrichter, Blood 70, 1245 (1987).

[27] The range $2 \lesssim \gamma \lesssim 4$ captures the sharpness of the observed transition and gives similar values of $\alpha$ and $\beta$. 